\title{
Simulation of laser-Compton cooling of electron beams for future linear colliders
}

\author{
T. Ohgaki ${ }^{1,2}$ and I. Endo ${ }^{3}$ \\ ${ }^{1}$ Lawrence Berkeley National Laboratory, Berkeley, California 94720 \\ ${ }^{2}$ Venture Business Laboratory, Hiroshima University, 2-313 Kagamiyama, Higashi-Hiroshima 739-8527, Japan \\ ${ }^{3}$ Graduate School of Advanced Sciences of Matter, Hiroshima University, 1-3-1 Kagamiyama, Higashi-Hiroshima 739-8530, Japan
}

(Received 2 October 2001; published 27 November 2001)

\begin{abstract}
We study a method of laser-Compton cooling of electron beams for future linear colliders. Using a Monte Carlo code, we evaluate the effects of the laser-electron interaction for transverse cooling. The optics with and without chromatic correction for the cooling are examined. The laser-Compton cooling for Japan Linear Collider/Next Linear Collider at $E_{0}=2 \mathrm{GeV}$ is considered.
\end{abstract}

DOI: $10.1103 /$ PhysRevSTAB.4.111001

\section{INTRODUCTION}

An operation of the $e^{+} e^{-}$linear collider at a center-ofmass energy of 0.5 to $5 \mathrm{TeV}$ requires a luminosity in excess of $10^{34} \mathrm{~cm}^{-2} \mathrm{~s}^{-1}$ for a study of particle physics. To achieve the required luminosity in several $\mathrm{TeV}$ colliders, the phase space of the electron and positron beams must be significantly reduced before the beam is accelerated in a main linear accelerator.

The technique which could accomplish the required cooling for the linear colliders was proposed by Palmer and Telnov [1,2] and is laser-Compton cooling. In laserCompton cooling, the beam loses both transverse and longitudinal momentum by Compton scattered photons, during head-on collisions with laser photons. The longitudinal momentum is restored to the beam in a linear accelerator. Since the Compton scattered photons follow the initial electron trajectory with a small additional spread due to much lower energy of photons (a few $\mathrm{eV}$ ) than the energy of electrons (several $\mathrm{GeV}$ ), the transverse distribution of electron beams remains almost unchanged and also the angular spread is almost constant. Consequently the emittance $\epsilon_{i}=\sigma_{i} \sigma_{i}^{\prime}$ remains almost unchanged $(i=x, y)$, where $\sigma_{i}, \sigma_{i}^{\prime}$ are the transverse beam size and the angular divergence. At the same time, the electron energy decreases from $E_{0}$ to $E_{f}$. Thus the normalized emittances have decreased as follows:

$$
\epsilon_{n}=\gamma \epsilon=\epsilon_{n 0}\left(E_{f} / E_{0}\right)=\epsilon_{n 0} / C,
$$

where $\epsilon_{n 0}, \epsilon_{n}$ are the initial and final normalized emittances, $\gamma=E / m_{e} c^{2}, m_{e}$ is electron mass, and the factor of the emittance reduction $C=E_{0} / E_{f}$. The method of electron beam cooling, repeated many times, allows further reduction of the transverse emittances after damping rings or guns by 1-3 orders of magnitude [2].

In this paper, we evaluated the effects of the laserCompton interaction for transverse cooling using the Monte Carlo code [3,4]. The simulation calculates the effects of the nonlinear Compton scattering between the laser photons and the electrons during a multicooling stage. Next, we examine the optics for cooling with and without
PACS numbers: 41.75.Fr, 29.17.+w, 41.85.Gy, 13.88.+e

chromatic correction. The laser-Compton cooling for Japan Linear Collider/Next Linear Collider (JLC/NLC) [5] at $E_{0}=2 \mathrm{GeV}$ is considered in Sec. IV. A summary of our conclusion is given in Sec. V.

\section{LASER-ELECTRON INTERACTION}

\section{A. Laser-electron interaction}

In this section we describe the main parameters for laser-Compton cooling of electron beams. A laser photon of energy $\omega_{L}$ (wavelength $\lambda_{L}$ ) is backward-Compton scattered by an electron beam of energy $E_{0}$ in the interaction point (IP). The kinematics of Compton scattering is characterized by the dimensionless parameter [2]

$$
x_{0} \equiv \frac{4 E_{0} \omega_{L}}{m_{e}^{2} c^{4}}=0.019 \frac{E_{0}[\mathrm{GeV}]}{\lambda_{L}[\mu \mathrm{m}]} .
$$

The parameters of the electron and laser beams in laserCompton cooling are listed in Tables I and II. The parameters of the electron beam with $2 \mathrm{GeV}$ are given for the JLC/NLC case in Sec. IV. The parameters of the electron beams with $5 \mathrm{GeV}$ are used for simulation in the next subsection. The wavelength of the laser is assumed to be $0.5 \mu \mathrm{m}$. The parameters of $x_{0}$ with the electron energies 2 and $5 \mathrm{GeV}$ are 0.076 and 0.19 , respectively.

The required laser flush energy with $Z_{R} \ll l_{\gamma} \simeq l_{e}$ is [2]

$$
A=25 \frac{l_{e}[\mathrm{~mm}] \lambda_{L}[\mu \mathrm{m}]}{E_{0}[\mathrm{GeV}]}(C-1)[\mathrm{J}],
$$

where $Z_{R}, l_{\gamma}\left(\sim 2 \sigma_{L, z}\right)$, and $l_{e}\left(\sim 2 \sigma_{z}\right)$ are the Rayleigh length of the laser and the bunch lengths of laser and electron beams, respectively. From this formula, the parameters of $A$ with the electron energies 2 and $5 \mathrm{GeV}$ are 56 and $4 \mathrm{~J}$, respectively.

The nonlinear parameter of the laser field is [2]

$$
\xi^{2}=4.3 \frac{\lambda_{L}^{2}\left[\mu \mathrm{m}^{2}\right]}{l_{e}[\mathrm{~mm}] E_{0}[\mathrm{GeV}]}(C-1) .
$$

In this study, for the electron energies 2 and $5 \mathrm{GeV}$, the parameters of $\xi$ are 2.2 and 1.5 , respectively. 
TABLE I. Parameters of the electron beams in laser-Compton cooling. The value in the parentheses is given by Telnov's formulas.

\begin{tabular}{ccccccc}
\hline \hline$E_{0}(\mathrm{GeV})$ & $E_{f}(\mathrm{GeV})$ & $C$ & $\epsilon_{n, x} / \epsilon_{n, y}(\mathrm{~m} \mathrm{rad})$ & $\beta_{x} / \beta_{y}(\mathrm{~mm})$ & $\sigma_{z}(\mathrm{~mm})$ & $\delta(\%)$ \\
\hline 2 & 0.2 & 10 & $7.4 \times 10^{-8} / 2.9 \times 10^{-8}$ & $4 / 4$ & 0.5 & $11(9.8)$ \\
5 & 1 & 5 & $3.0 \times 10^{-6} / 3.0 \times 10^{-6}$ & $0.1 / 0.1$ & 0.2 & $19(19)$ \\
\hline \hline
\end{tabular}

TABLE II. Parameters of the laser beams for laser-Compton cooling. The value in the parentheses is given by Telnov's formulas.

\begin{tabular}{ccccccc}
\hline \hline$E_{0}(\mathrm{GeV})$ & $\lambda_{L}(\mu \mathrm{m})$ & $x_{0}$ & $A(\mathrm{~J})$ & $\xi$ & $R_{L, x} / R_{L, y}(\mathrm{~mm})$ & $\sigma_{L, z}(\mathrm{~mm})$ \\
\hline 2 & 0.5 & 0.076 & $300(56)$ & $1.9(2.2)$ & $0.3 / 0.3$ & 1.25 \\
5 & 0.5 & 0.19 & $20(4)$ & $1.5(1.5)$ & $0.1 / 0.1$ & 0.4 \\
\hline \hline
\end{tabular}

The rms energy of the electron beam after Compton scattering is [2]

$$
\begin{array}{r}
\sigma_{e}=\frac{1}{C^{2}}\left[\sigma_{e 0}^{2}\left(\mathrm{GeV}^{2}\right)+0.7 x_{0}(1+0.45 \xi)\right. \\
\left.\times(C-1) E_{0}^{2}\left(\mathrm{GeV}^{2}\right)\right]^{1 / 2}(\mathrm{GeV}),
\end{array}
$$

where the rms energy of the initial beam is $\sigma_{e 0}$ and the ratio of energy spread is defined as $\delta=\sigma_{e} / E_{f}$. If the parameter $\xi$ or $x_{0}$ is larger, the energy spread after Compton scattering is increasing and it is the origin of the emittance growth in the defocusing optics, reacceleration linac, and focusing optics. The energy spreads $\delta$ for the electron energies 2 and $5 \mathrm{GeV}$ are $9.8 \%$ and $19 \%$, respectively.

The equilibrium emittances due to Compton scattering for the case of circular polarization are

$$
\begin{array}{r}
\epsilon_{n i, \min }=\frac{7.3 \times 10^{-10} \beta_{i}[\mathrm{~mm}]}{\lambda_{L}[\mu \mathrm{m}]}\left(1+\xi^{3}\right) \\
\quad(i=x, y)[\mathrm{m} \mathrm{rad}],
\end{array}
$$

where $\beta_{i}$ are the beta functions at IP. From this formula we can see that small beta gives small emittance. However, the large change of the beta functions between the magnet and the IP causes the emittance growth. Taking no account of the emittance growth, for the electron energies 2 and $5 \mathrm{GeV}$, the equilibrium emittances are $4.4 \times 10^{-8} \mathrm{~m} \mathrm{rad}$ and $6.2 \times 10^{-10} \mathrm{~m} \mathrm{rad}$, respectively.

\section{B. Simulation of laser-electron interaction}

For the simulation of laser-electron interaction, the electron beam is simply assumed to be a round beam in the case of $E_{0}=5 \mathrm{GeV}$ and $C=5$. Taking no account of the emittance growth of optics, the one stage for cooling consists of two parts as follows: (i) the laser-Compton interaction between the electron and laser beams and (ii) the reacceleration of electrons in the linac.

In the first part, we simulated the interactions by the CAIN code [4]. This simulation calculates the effects of the nonlinear Compton scattering between the laser photons and the electrons. We assumed that the laser pulse interacts with the electron bunch in head-on collisions. The $\beta_{x}$ and $\beta_{y}$ at the IP are fixed to be $0.1 \mathrm{~mm}$. The initial energy spread of the electron beams is $1 \%$. The energy of laser pulse is $20 \mathrm{~J}$. There is 5 times difference in flash energy between the simulation and the prediction in Ref. [2], because the prediction in Ref. [2] gives minimum flash energy corresponding to the case when laser and electron beams have equal lengths and uniform longitudinal distributions, while in this paper the beams are Gaussian and $\sigma_{L, z} \approx 2 \sigma_{z}$. The polarization of the electron and laser beams are $P_{e}=1.0$ and $P_{L}=1.0$ (circular polarization), respectively. When the $x_{0}$ parameter is small, the spectrum of the scattered photons does not largely depend on the polarization combination. In order to accelerate the electron beams to $5 \mathrm{GeV}$ for recovery of energy in the second part, we simply added the energy $\Delta E=5 \mathrm{GeV}-$ $E_{\text {avg }}$ for reacceleration, where $E_{\text {avg }}$ is the average energy
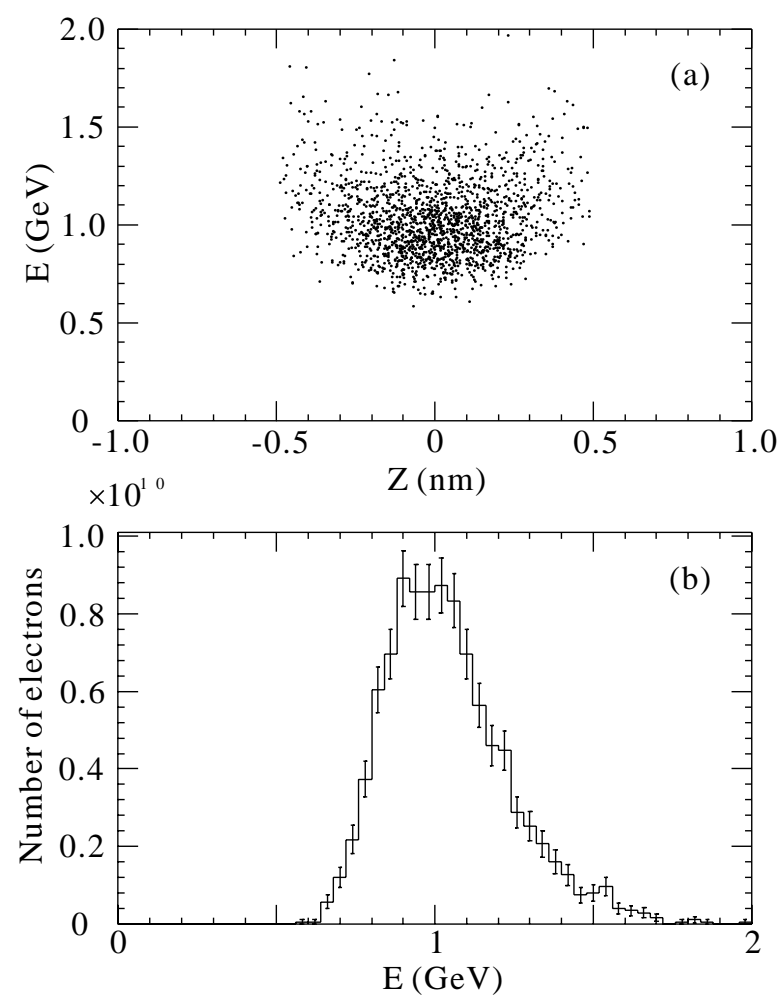

FIG. 1. The longitudinal distribution of the electrons. (a) The energy vs $z$. (b) The energy distribution of the electrons. The bin size is $40 \mathrm{MeV}$. 


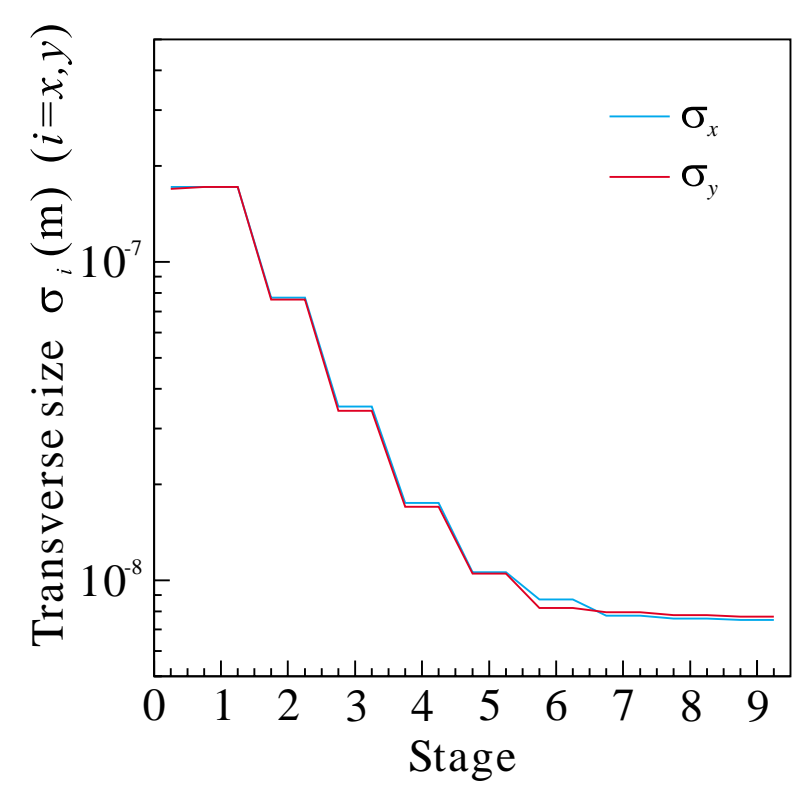

FIG. 2. (Color) The transverse sizes of the electron beams.

of the scattered electron beams after the laser-Compton interaction.

Figure 1 shows the longitudinal distribution of the electrons after the first laser-Compton scattering. The average energy of the electron beams is $1.0 \mathrm{GeV}$ and the energy spread $\delta$ is 0.19 . The longitudinal distribution seems to be a boomerang. If we assume a short Rayleigh length of laser pulse, the energy loss of the head and the tail of beams is small. The number of the scattered photons per incoming particle and the photon energy at the first stage are 40 and $96 \mathrm{MeV}$ (rms energy $140 \mathrm{MeV}$ ), respectively.

The transverse sizes of the electron beams in the multistage cooling are shown in Fig. 2. During collisions with the laser photons, the transverse distribution of the electrons remains almost unchanged. But they decrease when

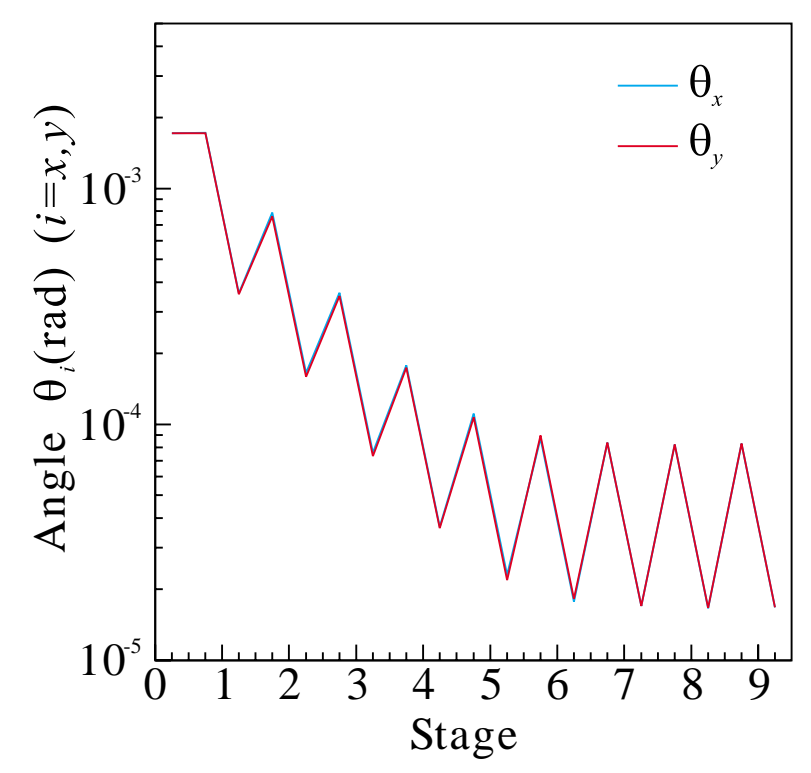

FIG. 3. (Color) The angles of the electron beams.

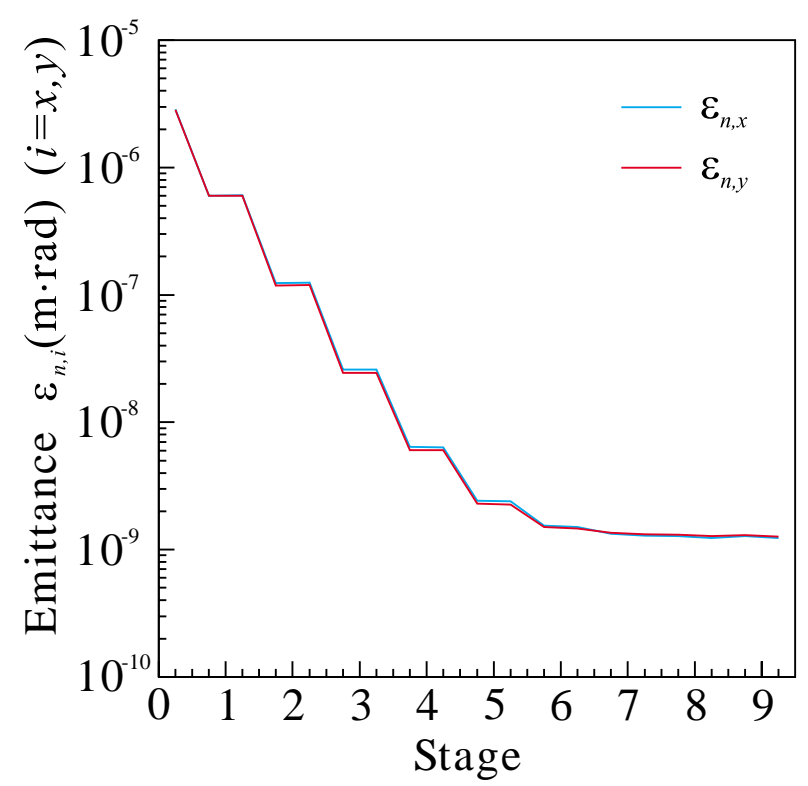

FIG. 4. (Color) The transverse emittances of the electron beams.

we focus them for the next laser-Compton interaction due to the lower normalized emittance and the fixed $\beta$ function at IP $\left(\sigma_{i}=\sqrt{\beta_{i} \epsilon_{n, i} / \gamma}\right)$. The angles of the electron beams in the multistage cooling, $\left.\theta_{i}=\sqrt{\left\langle\theta_{s, i}^{2}\right.}\right\rangle$, where $\theta_{s, i}$ is the scattering angle of the electron, are shown in Fig. 3 . As a result of reacceleration, the angles of the electrons decrease. They increase when we focus them for the next laser-Compton interaction. Finally, the angles attain the average of Compton scattering angle and the effect of cooling saturates.

Figure 4 shows the transverse emittances of the electron beams in the multistage cooling. From Eq. (6), $\epsilon_{n i, \min }=$ $6.2 \times 10^{-10} \mathrm{~m} \mathrm{rad}$, and the simulation presents $\epsilon_{n i, \min }=$

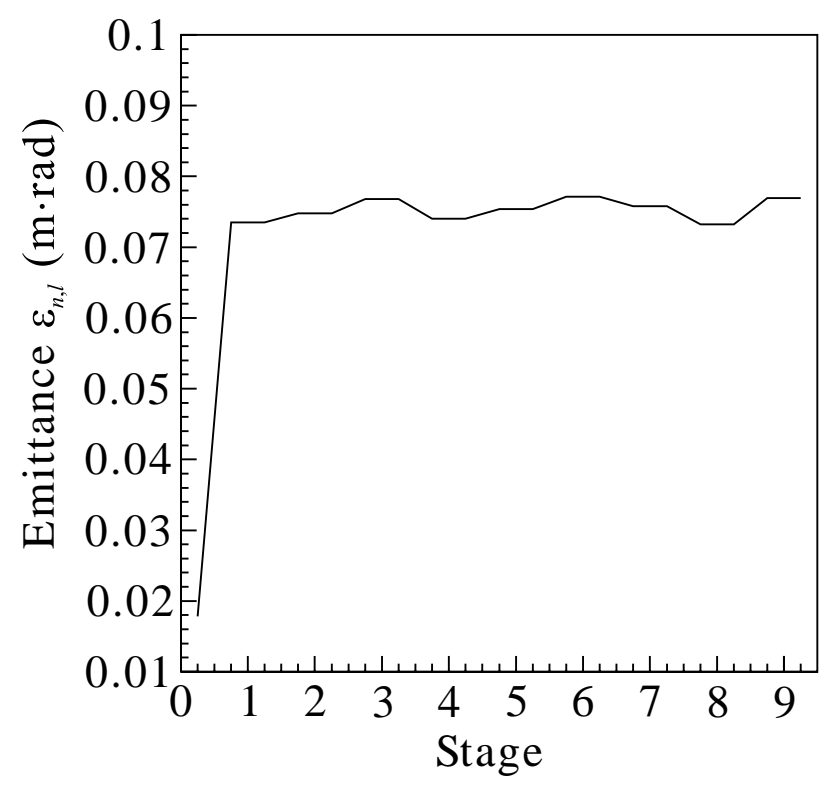

FIG. 5. The longitudinal emittance of the electron beams. 


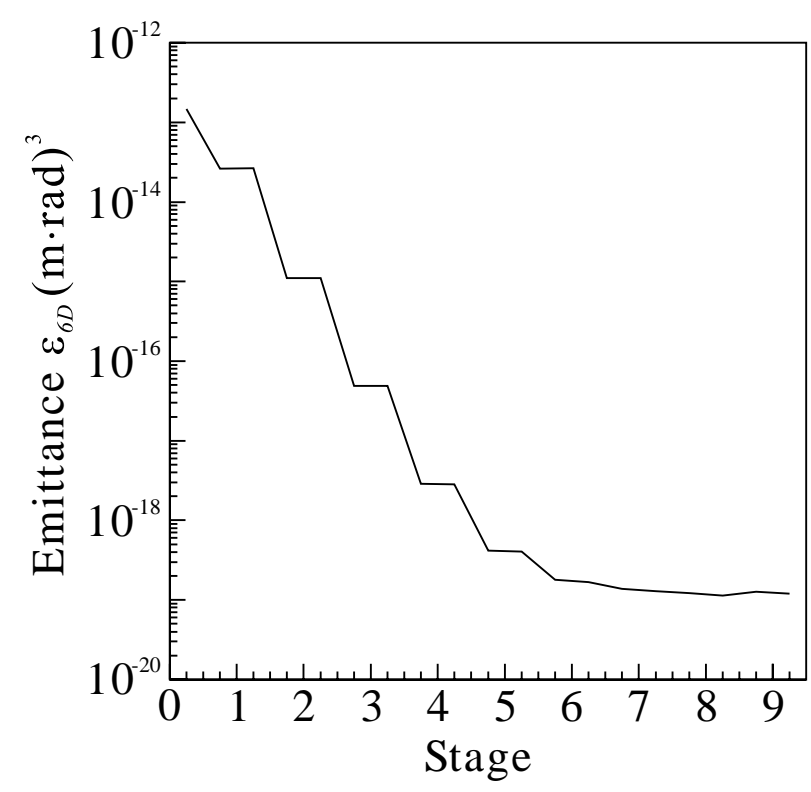

FIG. 6. The 6D emittance of the electron beams.

$1.2 \times 10^{-9} \mathrm{mrad}$. Figure 5 shows the longitudinal emittance of the electron beams in the multistage cooling. Because of the increase of the energy spread of the electron beams from $1 \%$ to $19 \%$, the longitudinal emittance rapidly increases at the first stage. After the first stage, the normalized longitudinal emittance is stable. The 6D emittance of the electron beams in the multistage cooling is shown in Fig. 6. The second cooling stage has the largest reduction for cooling. The eighth or ninth cooling stages have small reduction for cooling. The initial and final $6 \mathrm{D}$ emittances $\epsilon_{6 N}$ are $1.5 \times 10^{-13}(\mathrm{~m} \mathrm{rad})^{3}$ and $1.2 \times 10^{-19}(\mathrm{~m} \mathrm{rad})^{3}$, respectively.

Figure 7 shows the polarization of the electron beams in the multistage cooling. The decrease of the polarization

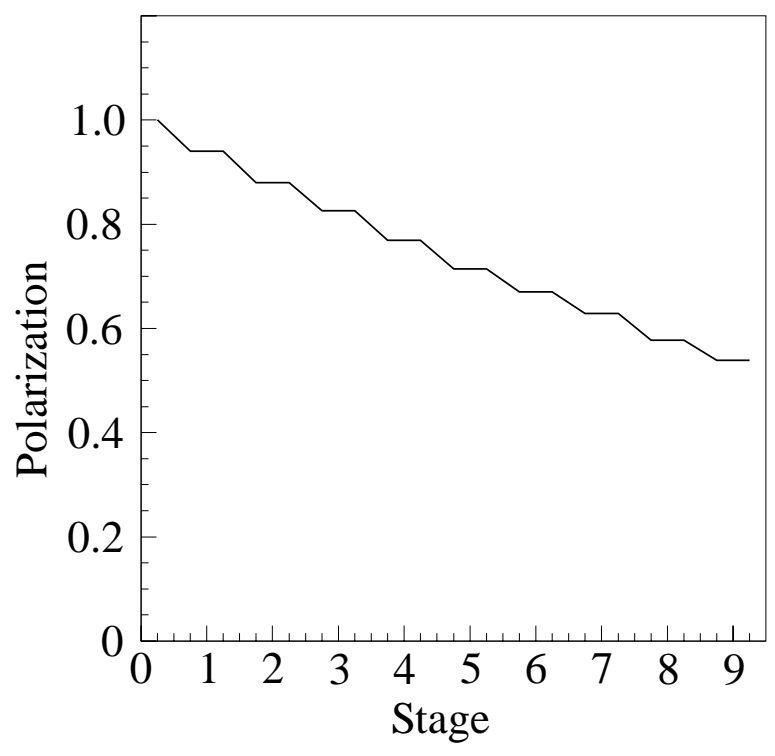

FIG. 7. The polarization of the electron beams. during the first stage is about 0.06. After six stages which the emittances come to their minimum value, the final polarization $P_{e}$ at the sixth stage is 0.67 .

\section{OPTICS DESIGN FOR LASER-COMPTON COOLING}

\section{A. Optics without chromaticity correction}

There are three optical devices for the laser-Compton cooling of electron beams as follows: (i) the focusing optics to the first IP, (ii) the defocusing optics from the first IP to the reacceleration linac, and (iii) the focusing optics from the linac to the next IP.

Figure 8 shows a schematic diagram of the laserCompton cooling of the electron beams. Optics (i) is focusing the electron beams from a few meters of $\beta$ function to several millimeters in order to effectively interact them with the laser beams. Optics (ii) is defocusing them from several millimeters to a few meters for reacceleration of electron beams in a linac. In a multistage cooling system, optics (iii) is needed for cooling in the next stage. The key problem for the focusing and defocusing optical devices is that the energy spread of electrons and the electron beams with a large energy spread are necessary to minimize or correct the chromatic aberrations avoiding emittance growth.

In this subsection we discuss the optics for laserCompton cooling without chromatic corrections. For the focusing and defocusing of the beams, we use the final doublet system which is similar to that of the final focus system of the future linear colliders [5]. The pole-tip field of the final quadrupole $B_{T}$ is limited to $1.2 \mathrm{~T}$ and the poletip radius $a$ is greater than $3 \mathrm{~mm}$. The strength of the final quadrupole is

$$
\kappa=B_{T} /(a B \rho) \leq 120 / E[\mathrm{GeV}]\left[\mathrm{m}^{-2}\right],
$$

where $B, \rho$, and $E$ are the magnetic field, the radius of curvature, and the energy of the electron beams. In our case, the electron energies in the optics (i), (ii), and (iii) are 5.0, 1.0, and $5.0 \mathrm{GeV}$, respectively, and the limit of the strength of the quadrupole in laser cooling is much larger than that of the final quadrupole of the future linear colliders. Because of the low energy beams in laser cooling,

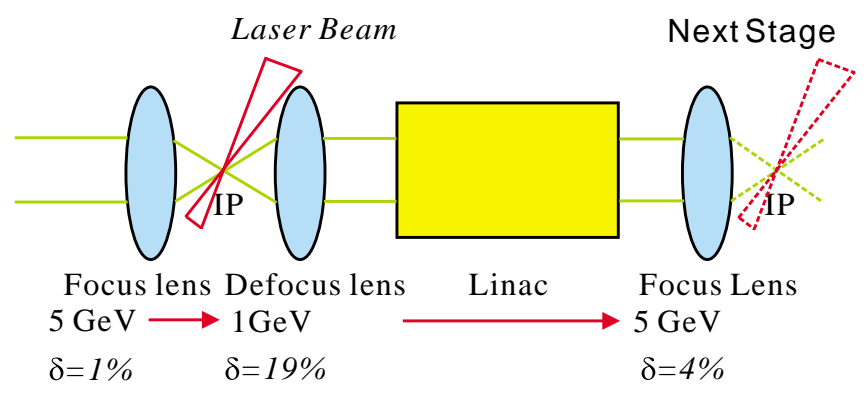

FIG. 8. (Color) Schematic diagram of the laser-Compton cooling of the electron beams. 


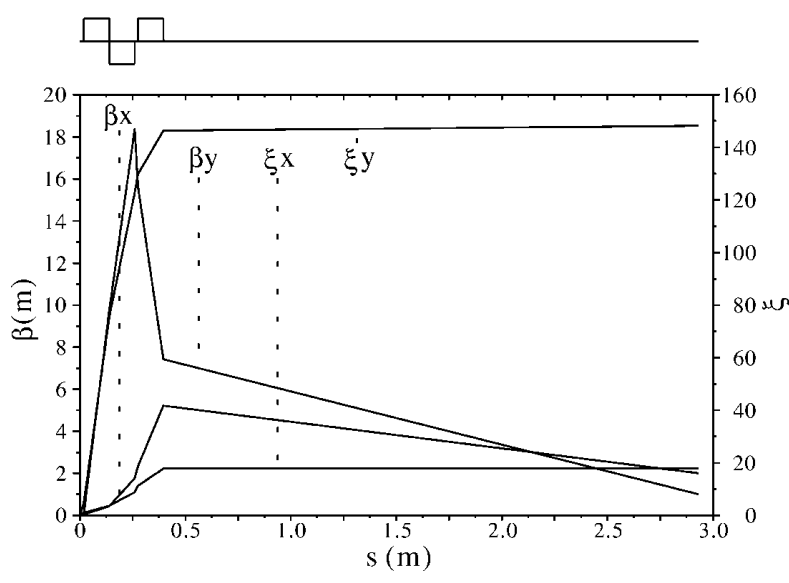

FIG. 9. The defocusing optics without chromaticity correction for laser-Compton cooling.

the synchrotron radiation from quadrupoles and bends is negligible.

The difference between the three optical devices is the amount of the energy spread of the beams. In the optics (i), (ii), and (iii), the beams have one, several tens, and a few percent energy spread. In order to minimize the chromatic aberrations, we need to shorten the length between the final quadrupole and the IP. In this study, the length from the face of the final quadrupole to the IP $l$ is assumed to be $2 \mathrm{~cm}$. Here we estimated the emittance growth in optics (ii), because the chromatic effect in optics (ii) is the most serious. Figure 9 shows the defocusing optics for laserCompton cooling by the MAD code [6]. The input file is attached to Ref. [7]. The parameters of the electron beam for laser-Compton cooling at $E_{0}=5 \mathrm{GeV}$ and $C=$ 5 are listed in Table III. The initial $\beta_{x}$ and $\beta_{y}$ after laserCompton interaction are 20 and $4 \mathrm{~mm}$, respectively. The final $\beta_{x}$ and $\beta_{y}$ are assumed to be 2 and $1 \mathrm{~m}$, respectively. The initial and final $\alpha_{x(y)}$ with no energy spread $\delta=0$ are 0 in this optics. The strength $\kappa$ of the final quadrupole for the beam energy of $1 \mathrm{GeV}$ from Eq. (7) is assumed to be $120 \mathrm{~m}^{-2}$.

In our case, the chromatic functions $\xi_{x}$ and $\xi_{y}$ are 18 and 148, respectively. The dependence of the emittances in the defocusing optics without chromaticity correction on the relative energy spread is shown in Fig. 10. In Ref. [8], the analytical study by thin-lens approximation has been studied for the focusing system, and here the transverse emittances are calculated by a particle-tracking simulation. The 10000 particles are tracked for the transverse and longitudinal Gaussian distribution by the MAD code. The relative energy spread $\delta$ is changed from 0 to 0.4 . Because
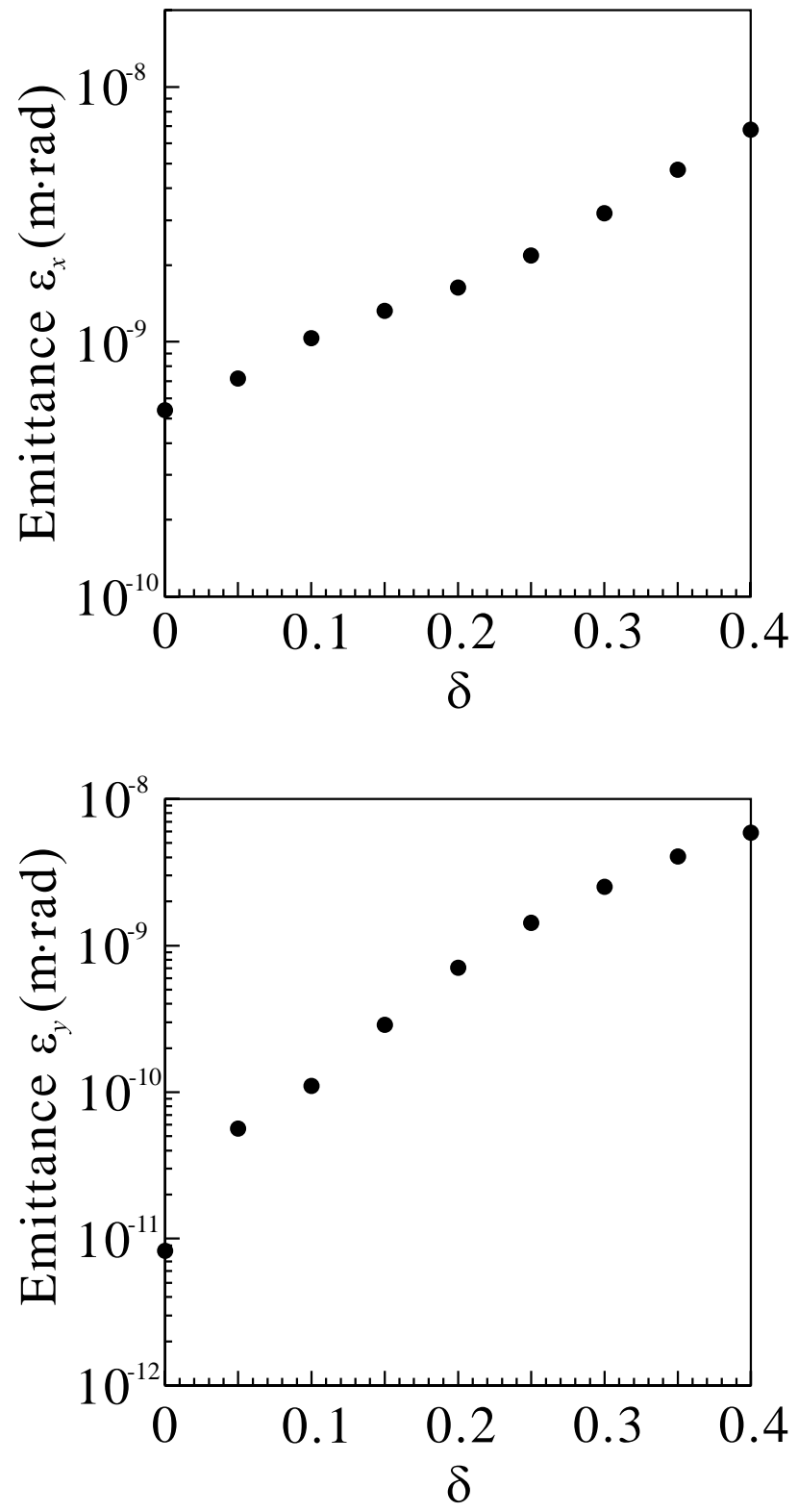

FIG. 10. Dependence of the emittances in the defocusing optics without chromaticity correction on the relative energy spread.

of the larger chromaticity $\xi_{y}$, the emittance $\epsilon_{y}$ is rapidly increasing with the energy spread $\delta$. If we set a limit of $200 \%$ for $\Delta \epsilon_{i} / \epsilon_{i}(i=x, y)$, the permissible energy spread $\delta_{x}$ and $\delta_{y}$ are 0.11 and 0.012 which mean the momentum bandwidth $\pm 22 \%$ and $\pm 2.4 \%$, respectively. The results are not sufficient for cooling at $E_{0}=5 \mathrm{GeV}$ and $C=5$, because the beams through the defocusing optics have the

TABLE III. Parameters of the electron beam for laser-Compton cooling at $E_{0}=5 \mathrm{GeV}$ and $C=5$ for the optics design.

\begin{tabular}{ccccc}
\hline \hline$E_{0}(\mathrm{GeV})$ & $\epsilon_{n, x} / \epsilon_{n, y}(\mathrm{~m} \mathrm{rad})$ & $\beta_{x} / \beta_{y}(\mathrm{~mm})$ & $\sigma_{x} / \sigma_{y}(\mathrm{~m})$ & $\sigma_{z}(\mathrm{~mm})$ \\
\hline 5 & $1.06 \times 10^{-6} / 1.6 \times 10^{-8}$ & $20 / 4$ & $3.3 \times 10^{-5} / 1.8 \times 10^{-7}$ & 0.2 \\
\hline \hline
\end{tabular}


energy spread of several tens percent. On the one hand, the optics can be useful as optics (i) and (iii) with the energy spread of a few percent.

\section{B. Optics with chromaticity correction}

The optics without chromaticity correction for optics (ii) does not work as we saw in the previous subsection. In this subsection we apply the chromaticity correction for optics (ii). The lattice for cooling is designed referring to the final focusing system of the future linear colliders by Oide [9]. The final doublet system is the same lattice as the optics in the previous subsection. The method of chromaticity correction uses one family of sextupole to correct for vertical chromaticity and, moreover, we added two weak sextupoles in the lattice to correct for horizontal chromaticity. Figure 11 shows the defocusing optics with chromaticity correction for laser-Compton cooling. The input file is attached to Ref. [7]. The total length of the lattice is about $63 \mathrm{~m}$.

The dependence of the emittances in the defocusing optics with chromaticity correction on the relative energy spread is shown in Fig. 12. The 10000 particles are tracked for the transverse and longitudinal Gaussian distribution by the MAD code. The relative energy spread $\delta$ is changed from 0 to 0.06 with the conservation $\kappa_{2} \theta_{B}$, where $\kappa_{2}$ and $\theta_{B}$ are the strength of the sextupole and the

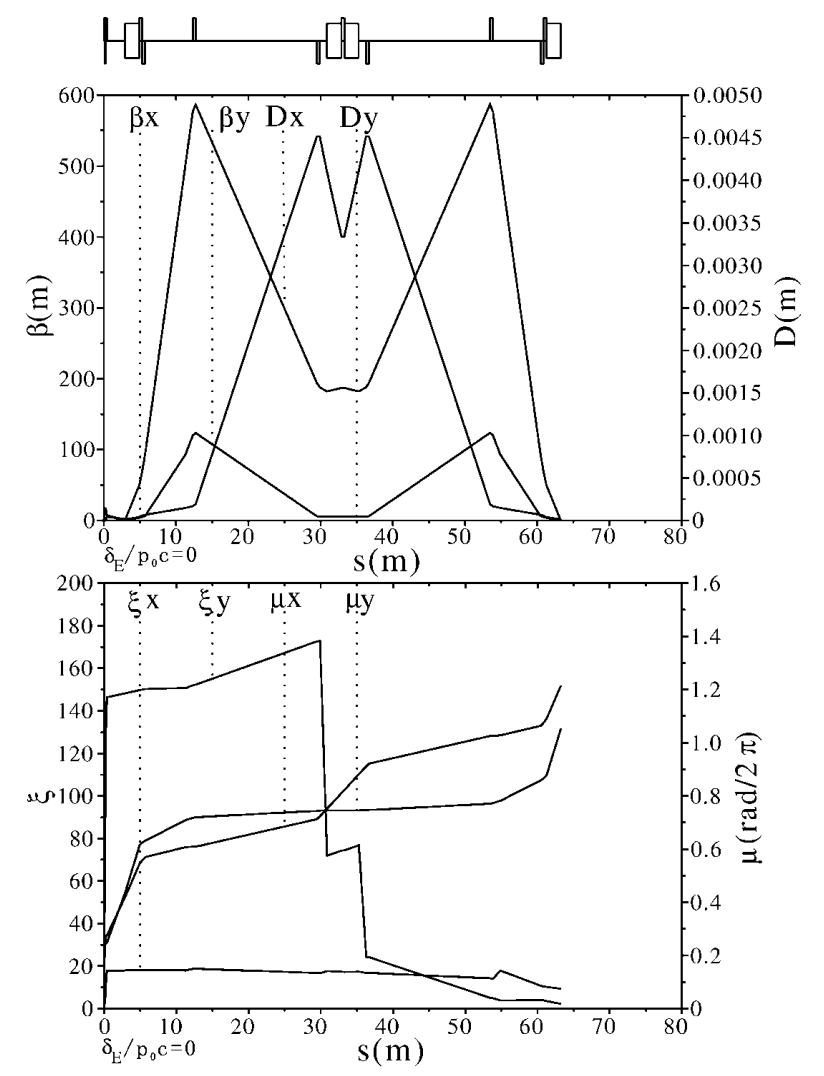

FIG. 11. The defocusing optics with chromaticity correction for laser-Compton cooling. angle of the bending magnet. The initial $\beta_{x}$ and $\beta_{y}$ after laser-Compton interaction are 20 and $4 \mathrm{~mm}$, respectively. The final $\beta_{x}$ and $\beta_{y}$ are assumed to be 2 and $1 \mathrm{~m}$, respectively. The initial and final $\alpha_{x}(y)$ with no energy spread $\delta=0$ are 0 in this optics. After the chromaticity correction, the chromaticity functions $\xi_{x}$ and $\xi_{y}$ are 9.3 and 1.6, respectively. If we set a limit of $200 \%$ for $\Delta \epsilon_{i} / \epsilon_{i}$ $(i=x, y)$, the permissible energy spread $\delta_{x}$ and $\delta_{y}$ are 0.040 and 0.023 which mean the momentum band width $\pm 8 \%$ and $\pm 4.6 \%$, respectively. By comparison with the results of the optics with chromaticity correction at a limit of $200 \%$ for $\Delta \epsilon_{i} / \epsilon_{i}(i=x, y)$, the $\epsilon_{y}$ of the optics without chromaticity correction is about 2 times larger than that of the optics with chromaticity correction, but the $\epsilon_{x}$ of the optics with chromaticity correction is 3 times smaller than that of the one before. The results are still not sufficient
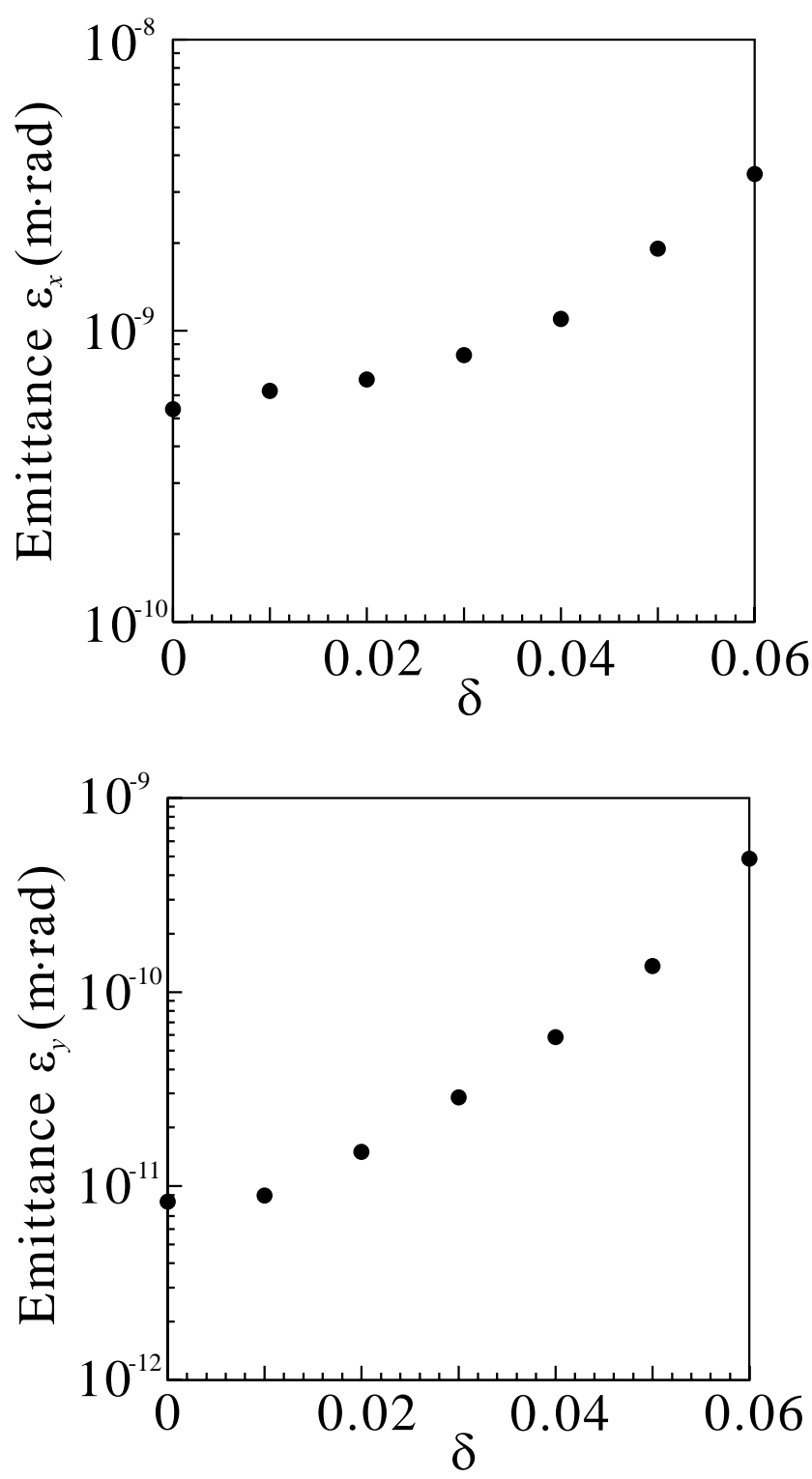

FIG. 12. Dependence of the emittances in the defocusing optics with chromaticity correction on the relative energy spread. 
for cooling with $E_{0}=5 \mathrm{GeV}$ and $C=5$. These results emphasize the need to pursue further ideas for a plasma lens [10].

\section{LASER-COMPTON COOLING FOR JLC/NLC AT $E_{0}=2 \mathrm{GeV}$}

\section{A. Optics without chromaticity correction}

For the future linear colliders, the method of laserCompton cooling is effective to reduce the transverse emittances after damping rings. Where can it be placed? There are two possibilities for JLC/NLC [11] as follows: (i) after the first bunch compressor $(\mathrm{BC} 1)$ and before the prelinac, $E_{0}=2 \mathrm{GeV}$ and $\sigma_{z}=0.5 \mathrm{~mm}$; (ii) after the second compressor (BC2) and before the main linac, $E_{0}=10 \mathrm{GeV}$ and $\sigma_{z}=0.1 \mathrm{~mm}$.

Case (ii) needs a large energy for recovery after Compton scattering, and we consider case (i) in this study. The parameters of the electron and laser beams for laserCompton cooling for JLC/NLC at $E_{0}=2 \mathrm{GeV}$ and $C=$ 10 are listed in Tables I and II. The energy of the laser pulse is $300 \mathrm{~J}$. The initial energy spread of the electron beams is $1 \%$. The simulation results of the laser-electron interaction by the CAIN code are summarized as follows. The energy spread of the electron beam is $11 \%$. The decrease of the longitudinal polarization of the electron beam is $0.038\left(P_{e}=1.0, P_{L}=1.0\right)$. The number of the scattered photons per incoming particle and the photon energy are 200 and $8.9 \mathrm{MeV}$ (rms energy $19 \mathrm{MeV}$ ), respectively.

The electron energy after Compton scattering in case (ii) is $0.2 \mathrm{GeV}$, and the strength of the final quadrupole from Eq. (7) is $600 \mathrm{~m}^{-2}$. Table IV lists the parameters of the defocusing optics for laser-Compton cooling for JLC/NLC at $E_{0}=2 \mathrm{GeV}$ and $C=10$. The final $\beta_{x}$ and $\beta_{y}$ are assumed to be 1 and $0.25 \mathrm{~m}$, respectively. The chromaticity functions $\xi_{x}$ and $\xi_{y}$ are 18 and 23, respectively. Using the MAD code, the emittance growth in the defocusing optics is

$$
\begin{aligned}
\Delta \epsilon_{n, x}^{\text {defocus }} & =\epsilon_{n, x}-\epsilon_{n, x 0} \\
& \sim 1.0 \epsilon_{n, x 0} \sim 7.6 \times 10^{-8}[\mathrm{~m} \mathrm{rad}], \\
\Delta \epsilon_{n, y}^{\text {defocus }} & =\epsilon_{n, y}-\epsilon_{n, y 0} \\
& \sim 1.6 \epsilon_{n, y 0} \sim 4.6 \times 10^{-8}[\mathrm{~m} \mathrm{rad}],
\end{aligned}
$$

where the normalized emittances before and after the defocusing optics are $\epsilon_{n, i 0}$ and $\epsilon_{n, i}(i=x, y)$, respectively.

TABLE IV. Parameters of the defocusing optics for laser-Compton cooling for JLC/NLC at $E_{0}=2 \mathrm{GeV}$ and $C=10$.

\begin{tabular}{ccccc}
\hline \hline$l$ & $\begin{array}{c}\text { Length } \\
\text { of Q1 }\end{array}$ & Field of Q1 & Aperture & $\begin{array}{c}\text { Total } \\
\text { length }\end{array}$ \\
\hline $5 \mathrm{~mm}$ & $2 \mathrm{~cm}$ & $1.2 \mathrm{~T}$ & $0.5 \mathrm{~mm}$ & $7.4 \mathrm{~cm}$ \\
\hline \hline
\end{tabular}

The emittance growth in the other two-focus optics is negligible. For the JLC/NLC case at $E_{0}=2 \mathrm{GeV}$ the reduction factor $C$ is 10 , we can understand that the cooling is very effective from Eqs. (8) and (9).

\section{B. Reacceleration linac}

In the reacceleration linac, there are two major sources of the emittance increase [11] as follows: (i) the emittance growth due to the misalignment of the quadrupole magnet and the energy spread and (ii) the emittance growth due to the cavity misalignment.

The emittance growth due to these sources in the reacceleration linac is formulated by Yokoya [11]

$$
\begin{aligned}
& \Delta \epsilon_{n, x}^{\mathrm{linac}} \sim 0.32 \epsilon_{n, x 0} \sim 2.4 \times 10^{-8}[\mathrm{~m} \mathrm{rad}], \\
& \Delta \epsilon_{n, y}^{\mathrm{linac}} \sim 0.82 \epsilon_{n, y 0} \sim 2.4 \times 10^{-8}[\mathrm{~m} \mathrm{rad}] .
\end{aligned}
$$

The final emittance growth and the final emittance with $C=10$ are

$$
\begin{aligned}
\Delta \epsilon_{n, x} & \sim 1.3 \epsilon_{n, x 0} \sim 1.0 \times 10^{-7}[\mathrm{~m} \mathrm{rad}] \\
& \Rightarrow \epsilon_{n, x} \sim 0.23 \epsilon_{n, x 0}, \\
\Delta \epsilon_{n, y} & \sim 2.4 \epsilon_{n, y 0} \sim 7.0 \times 10^{-8}[\mathrm{~m} \mathrm{rad}] \\
& \Rightarrow \epsilon_{n, y} \sim 0.34 \epsilon_{n, y 0} .
\end{aligned}
$$

Because the reduction factor $C$ is 10 for the JLC/NLC case at $E_{0}=2 \mathrm{GeV}$, the energy spread after cooling is $1.1 \%$ with the initial energy spread $1 \%$. The total reduction factor of the 6D emittance of the laser-Compton cooling for JLC/NLC at $E_{0}=2 \mathrm{GeV}$ is about 13. The decrease of the polarization of the electron beam is 0.038 due to the laser-Compton interaction.

\section{SUMMARY}

We studied the method of laser-Compton cooling of electron beams for future linear colliders. The effects of the laser-Compton interaction for cooling were evaluated by the Monte Carlo simulation. From the simulation in the multistage cooling, we showed that the low emittance beams with $\epsilon_{6 N}=1.2 \times 10^{-19}(\mathrm{~m} \mathrm{rad})^{3}$ can be achieved in our beam parameters. We also examined the optics with and without chromatic correction for cooling, but the optics are not sufficient for cooling due to the large energy spread of the electron beams.

The laser-Compton cooling for JLC/NLC at $E_{0}=$ $2 \mathrm{GeV}$ and $C=10$ was considered. The total reduction factor of the $6 \mathrm{D}$ emittance of the laser-Compton cooling is about 13. The decrease of the polarization of the electron beam is 0.038 due to the laser-Compton interaction.

\section{ACKNOWLEDGMENTS}

We thank Y. Nosochkov, K. Oide, T. Takahashi, V. Telnov, M. Xie, and K. Yokoya for useful comments and 
discussions. This work was supported in part by the U.S. Department of Energy under Contract No. DE-AC0376 SF00098.

[1] R. Palmer, Nucl. Instrum. Methods Phys. Res., Sect. A 355, 150 (1994).

[2] V. Telnov, Phys. Rev. Lett. 78, 4757 (1997); 80, 2747 (1998); in Proceedings of the 15th Advanced ICFA Beam Dynamics Workshop on Quantum Aspects of Beam Physics, Monterey, CA, 1998 (BINP Report No. BUDKERINP-98-33, 1998); Nucl. Instrum. Methods Phys. Res., Sect. A 455, 80 (2000).

[3] T. Ohgaki, in Proceedings of the 3rd International Workshop on Electron-Electron Interactions at TeV Energies, Santa Cruz, CA, 1999 [Int. J. Mod. Phys. A 15, 2587 (2000)].

[4] P. Chen, T. Ohgaki, A. Spitkovsky, T. Takahashi, and K. Yokoya, Nucl. Instrum. Methods Phys. Res., Sect. A 397, 458 (1997).
[5] LBNL Report No. LBNL-PUB-5424, SLAC Report No. 474, 1996; KEK Report No. 97-1, 1997; DESY Report No. 97-048, CERN Report No. ECFA-97-182, 1997.

[6] H. Grote and F. C. Iselin, CERN Report No. SL-90-13-AP, 1996.

[7] T. Ohgaki, LBNL Report No. 44380, 1999.

[8] B. W. Montague and F. Ruggiero, CERN Report No. CLICNOTE-37, 1987.

[9] K. Oide, Nucl. Instrum. Methods Phys. Res., Sect. A 276, 427 (1989); in Proceedings of the DPF Summer Study on High Energy Physics in the 1990s, Snowmass, CO, 1988 (SLAC Report No. PUB-4806, 1988); in Proceedings of the 1st Workshop on the Japan Linear Collider (JLC), Tsukuba, Japan, 1989 (KEK Report No. 89-190, 1989).

[10] P. Chen, K. Oide, A. M. Sessler, and S. S. Yu, Phys. Rev. Lett. 64, 1231 (1990); in Proceedings of the Fourteenth International Conference on High Energy Accelerators, Tsukuba, 1989 (SLAC Report No. PUB-5060, 1989).

[11] K. Yokoya, Nucl. Instrum. Methods Phys. Res., Sect. A 455, 25 (2000). 